and colleagues to highlight risk and provide support. A culture of open discussion and transparency was implemented through provision of Welcome Packs including Care and Discharge Pathways details at point of entry to the service. Support was provided to patients with Discharge Packs including information about community services. This enabled a meaningful support model at the end of their recovery journey and an improved discharge process.

The team is now working with additional teams and administrative and clerical staff to improve safety. We hope to replicate this approach in our Trust.

\section{The introduction of electronic prescribing in the Orchard clinic-a QI project}

Hannah Sayeed ${ }^{1 \star}$ and Fionnbar Lenihan ${ }^{2}$

${ }^{1}$ ST6 Forensic Psychiatry, the Orchard clinic and ${ }^{2}$ St Bricin's Military Hospital

${ }^{\star}$ Corresponding author.

doi: 10.1192/bjo.2021.581

Aims. Hospital Electronic Prescribing and Medicines Administration (HEPMA) system successfully rolled out in July, 2020 at the Orchard clinic as the first site in NHS Lothian. The initial aim was to collect some "pre-HEPMA" and "postHEPMA" data to look at "staff's attitudes to a new IT system, and does that change with successful implementation of it?" in the form of a survey. In the light of the findings of the pre-HEPMA staff survey, it became the QI project (as above).

This aimed to look at both qualitative data; in the form of a staff attitudes survey towards a new IT system and quantitative data; to measure benefits of its implementation and to address issues raised by staff in the survey in the form of an audit in both pre and post HEPMA cycles.

Method. Two cycles were completed as follows:

1. Pre-HEPMA cycle in March, 2020:

Survey: Questionnaires asking question re- own IT skills, preparation and expectations of outcome of its implementation.

Audit: Measured time taken to write and re-write paper prescriptions. Proforma filled by staff to measure time taken to log on and other IT related issues.

2. Post-HEPMA cycle in October, 2020:

Survey: Replicated above questions re-own IT skills, support during and after launch, disaster recovery and views about actual outcome of its implementation.

Audit: Replicated to measure time taken to complete electronic prescriptions.

Result. 24 staff questionnaires returned in both cycles. Staff felt more confident in their own IT skills, training and hence competence to use HEPMA; more supported, more confident about contingency plans and HEPMA to be more beneficial than initially anticipated. Overall, actual perceived success of (91\%) compared to anticipated success (71\%).

The majority issues raised via the first survey were felt to be addressed. Time to log was on average

The audit showed a clear benefit in terms of clinical time saving, e.g. daily clinical time spent writing prescription reduced from an average 45 to 6 minutes with HEPMA.

The quality of documentation on the prescriptions remained unchanged.

Conclusion. Staff's attitude towards IT does change after successful implementation of a new IT system. But IT issues make the whole process laborious.

There was evident benefits realization with an electronic prescribing system compared to paper prescriptions.
I was awarded "Employee of the month" for this project which I also presented at the NHS Lothian grand round (>150 attendees) for dissemination and future replication.

\section{Improving access to mental health services for homeless people in Torbay}

Tom Scott-Gatty ${ }^{1 \star}$ and Tom Cant ${ }^{2}$

${ }^{1}$ Devon Partnership Trust, Livewell Southwest NHS Trust, Shekinah and ${ }^{2}$ Devon Partnership Trust

${ }^{\star}$ Corresponding author.

doi: 10.1192/bjo.2021.582

Aims. Devon continues to see increasing numbers of rough sleepers despite the "Everyone In" initiative and the South West region is now behind only London and the South East nationally. The interaction of homelessness and Mental Health is complex. Mental health problems and trauma contribute to people becoming homeless as well as homelessness itself causing or exacerbating existing problems, all complicated by high rates of substance use and poor physical health. Despite the desperate need in this population they often struggle to access mental health services which are not designed with their needs in mind. Their pattern of service use is primarily that of acute services when in crisis and disengagement in the community which results in high costs and poor outcomes.

Method. In July 2019 an outreach service was set up consisting of a psychiatry core trainee (Dr Tom Scott-Gatty) for half a day per week supervised by the Torbay North CMHT consultant (Dr Tom Cant) to seek opportunities to engage individuals in assessment and treatment and improve outcomes in this population. The service is primarily based at the homeless hostel in Torquay (Leonard Stocks Centre) for ease of access but is flexible about where patients are seen. Patients have been seen in various locations including medical wards, prison, on the street etc. The role includes close work and liaison with other professionals such as GPs, probation, charity sector, drug and alcohol etc. and this is integral to supporting the level of complexity seen in this population. Engagement, building relationships and trust are central to serving this vulnerable and marginalised population. Result. In January 2021 feedback forms were completed by 13 patients and 18 professionals who had used the service. Feedback was overwhelmingly positive with average overall score 9/10 from both patients and professionals. All patients reported feeling comfortable using the service and that mental health services are now easier to access. All respondents would like to see the service continue. A significant number of patients and professionals identified increasing the hours offered by the service as an area for improvement.

Conclusion. This service has succeeded in improving access to mental health services for homeless people in Torbay. The service is valued by both the people it serves and the professionals supporting them. Further improvement to the service could be achieved by expanding capacity. Funding has been identified from existing local authority budgets to add a CPN to the team to achieve this.

\section{QI project: Improvement in quality of Seclusion Medical Review}

Shumaila Shahbaz ${ }^{\star}$ and Richard Ward

Humber Teaching Foundation Trust

${ }^{*}$ Corresponding author.

doi: 10.1192/bjo.2021.584 
Aims. To establish the improvements in the quality of seclusion medical review after introducing a template to complete the review.

Background. The Mental Health Act - Code of Practice outlines the standards of patient care while in seclusion. It also emphasis that supportive engagement/observation schedules should be reviewed in person and continued at the point an episode of seclusion was initiated.

Furthermore, NICE also set up standards to monitor side effect profile while prescribing psychotropic for such patients and regular management review. It also gives importance to staff training to ensure these standards.

To improve the quality of the seclusion medical review, we completed an audit in July 2019 to ascertain whether medics are following Trust Policy.

We identified good results (above 90\%) in the following areas:

Time of seclusion review

Record keeping

Management plan

Good documentation of risk, mental state examination and physical health.

We also noticed that the following areas can be improved:

Prescribed Medications. (60\%)

Medication side effects. (40\%)

Physical Observations (40\%)

We used the following audit standards for our audit after our last audit and a template was designed and after discussion with medics incorporated into the existing documentation template.

Time of review

Reason and duration for seclusion

Psychiatric diagnosis

Mental State Examination/Behaviour

Physical health (including physical observations)/Environment

Medication (prescribed, rapid tranquilisation, side effects, or adverse effects)

Risk (to self-DSH or accidental) (risks to others)

Plan :(frequency of physical obs./medical review, management, restrictions, exit plan for terminating seclusion, patient's capacity to understand it)

Method. We considered the following aspects:

Retrospective data collection from 01.03.2020 to 30.08.2020.

Sample selection: random selection of mixture of clinicians on different times and days of the week.

Data analysis was carried out by using Microsoft Excel.

Result. We noticed a marked improvement in the quality of seclusion medical review (between 95\% and 100\%) after introducing a template for it. There were no major concerns identified during the re-audit.

Conclusion. To continue to use the template for Seclusion Medical Review which has shown significant improvement in the quality of the reviews which will improve patient care.

It also helped us to deliver person centred care and safe practice.

To continue teaching and training of doctors.

This QIP project motivated nurses to do an audit on nursing seclusion review and made necessary changes.

\section{Quality of seclusion medical review according to trust guidelines}

Shumaila Shahbaz ${ }^{*}$ and Richard Ward

Humber Teaching Foundation Trust

${ }^{\star}$ Corresponding author.

doi: 10.1192/bjo.2021.585
Aims. We accessed whether medics are following Trust Policy while conducting seclusion medical review and identify the strengths in quality of seclusion medical review and identify the areas which need improvements to improve our quality and standards of patient's care and safety and to reduce risks.

Background. The Mental Health Act Code of Practice sets an expectation for mental health services for restrictive interventions (use of restraint, seclusion and rapid tranquilisation) by following good standards. Medical reviews provide an opportunity to evaluate and amend seclusion management plan. This clinical audit was undertaken by looking at quality of record keeping about seclusion review by junior doctors, staff grades and consultants at different times (day, night, and weekend).

Method. Data analysis was carried out by using Microsoft Excel. The audit had Humber Teaching NHSFT approval. We assessed electronic healthcare records. Data collection was carried out or retrospectively in $2019(\mathrm{n}=40)$ using following parameters:

1) A review of patient's physical and psychiatric health.

2) An assessment medication prescribed and adverse effects of medication.

3) A review of observations required.

4) An assessment of the risk posed by the patient to others.

5) An assessment of any risk to the patient from deliberate or accidental self-harm.

6) An assessment of need for continuing seclusion, and whether it is possible for seclusion measures to be applied more flexibly, or in a less restrictive manner.

7) Time of Seclusion Review: Within first hour after seclusion and then every 4 hours until internal MDT. After MDT twice a day.

8) Record Keeping.

Result. Key Successes (above 80\%)

Time of seclusion review (with in first hour or when required)

Record keeping (accurate time and place for clinical notes).

Plan for continuing need for seclusion.

Good documentation of Risk to self and risk to others.

Good documentation of mental state examination.

Comments on physical health although it can be improved.

Key Concerns(Less than 60\%):

Prescribed Medications.

Medication side effects.

Physical Observations

Conclusion. Medics are missing some important parts in seclusion medical review. We developed a template for seclusion medical review according to trust guidelines which are based on Code of Practice and to incorporate in already existing seclusion review form. We also delivered teaching and training to doctors and also showed junior doctor's an example of documentation. We will re-audit in 1 years' time to see improvement.

Pilot project: easy read psychiatry clinic appointment outcome letters

Anu Sharma ${ }^{1 \star}$ and Indermeet Sawhney ${ }^{2}$

${ }^{1}$ Saffron Ground, Ditchmore lane and ${ }^{2}$ Tekhnicon House,

Springwood Drive

${ }^{\star}$ Corresponding author.

doi: 10.1192/bjo.2021.586

Aims. To improve communication with patients and carers by sharing information in an easily comprehensible manner. 